\title{
Emergency and on-demand health care: modelling a large complex system
}

\author{
SC Brailsford ${ }^{1 *}$, VA Lattimer ${ }^{2}, \mathrm{P}_{\text {Tarnaras }}{ }^{1}$ and JC Turnbull ${ }^{2}$ \\ ${ }^{1}$ University of Southampton, Southampton, UK; and ${ }^{2}$ School of Nursing and Midwifery, University of Southampton, \\ Southampton, $U K$
}

\begin{abstract}
This paper describes how system dynamics was used as a central part of a whole-system review of emergency and ondemand health care in Nottingham, England. Based on interviews with 30 key individuals across health and social care, a 'conceptual map' of the system was developed, showing potential patient pathways through the system. This was used to construct a stock-flow model, populated with current activity data, in order to simulate patient flows and to identify system bottle-necks. Without intervention, assuming current trends continue, Nottingham hospitals are unlikely to reach elective admission targets or achieve the government target of $82 \%$ bed occupancy. Admissions from general practice had the greatest influence on occupancy rates. Preventing a small number of emergency admissions in elderly patients showed a substantial effect, reducing bed occupancy by $1 \%$ per annum over 5 years. Modelling indicated a range of undesirable outcomes associated with continued growth in demand for emergency care, but also considerable potential to intervene to alleviate these problems, in particular by increasing the care options available in the community.
\end{abstract}

Journal of the Operational Research Society (2004) 55, 34-42. doi:10.1057/palgrave.jors.2601667

Keywords: emergency and on-demand health care; A\&E; simulation; system dynamics; discrete event simulation

\section{Introduction}

In this paper, we describe an application of system dynamics to a very large, complex system: the entire health-care system in the city of Nottingham, England, or to be more precise that part of it concerned with the delivery of emergency or 'unscheduled' care. The model was developed as part of a research project led by Dr Valerie Lattimer of the School of Nursing and Midwifery at the University of Southampton. This project, commissioned in 2001 by the (then) Nottingham Health Authority, was itself part of a larger, ongoing project in Nottingham, known as the Emergency Care-On Demand (ECOD) project. ${ }^{1}$ In Nottingham, emergency hospital admissions have risen dramatically in recent years. The ECOD project was designed to look at the whole healthcare system, to determine why demand is so high, and to investigate what could be done to alleviate this pressure. The Southampton contribution involved carrying out a system review and providing research support to the ECOD project.

Emergency or unscheduled care can be provided either in hospital (the secondary sector) or in the community (the primary sector). Many emergency hospital admissions occur as a result of patient visits to a hospital Accident and Emergency (A\&E) Department. Patients can also be admitted directly to the wards, usually as a result of a

*Correspondence: SC Brailsford, School of Management, University of Southampton, Southampton SO17 1BJ, UK.

E-mail: scb@soton.ac.uk referral by a General Practitioner (GP). In both cases, some patients may arrive by ambulance whereas others travel to hospital independently. A third group of emergency patients are admitted directly from outpatient clinics. In the community, unscheduled care is provided in a number of ways. In normal surgery hours, patients may request urgent or same-day GP appointments. After the surgery is closed, patients wishing to see a doctor urgently usually need to contact an out-of-hours GP service. This may be a cooperative of local GPs or a commercial deputizing service. Very few individual GPs now provide their own out-ofhours cover. Other services are available, including NHS Direct, a national 24/7 telephone help-line where people can seek medical advice and information. Staffed by nurses, who can seek medical opinion or summon an ambulance if necessary, this service was intended to enable people to make better decisions about accessing health care. Nottingham has a well-established NHS Direct, which is integrated with the largest GP cooperative Nottingham Emergency Medical Services (NEMS). Patients calling out of surgery hours simply dial the NHS Direct number, and if a doctor's visit is required, will be transferred directly through to NEMS and given an emergency appointment.

Another Government initiative, launched a couple of years after NHS Direct, was the introduction of 'Walk-in Centres', often located in shopping centres or supermarkets, where people can attend without an appointment. This is also a nurse-run system whose aim is to treat, or advise 
about, minor conditions, thus (in theory) freeing up the time of GPs and hospital A\&E Departments to deal with more serious cases. Nottingham has had a Walk-in Centre since June 2000. Other community services providing health advice or access to the health-care system include Social Services, pharmacist shops, the dental services, and community mental health teams. Finally, the '999' emergency services-Fire, Police and obviously the Ambulance Service-provide emergency care and access to the NHS system.

\section{Background to the problem}

Nottingham is a city of about 640000 inhabitants in the East Midlands of England. The city is served by two acute NHS Hospital Trusts, Queens Medical Centre (QMC) and Nottingham City Hospital (NCH). Both are teaching hospitals. NCH has approximately 1000 beds. Of 77230 admissions to NCH between April 2000 and March 2001, $25755(33 \%)$ were emergency admissions. QMC has 1441 beds, including approximately 1000 acute beds. QMC admitted 97850 patients in 2000-2001, of whom 37789 (39\%) were emergency admissions. QMC has the only A\&E department in the city. Outpatient attendances and inpatient admission rates are approximately three times the national average, although A\&E attendances decreased slightly $(1.5 \%)$ in 2000-2001 over the previous year. At both hospitals, there has been an increase in people needing emergency care for the past 3 years (see Table 1). The A\&E department at QMC is one of the busiest in England, seeing over 120000 patients between April 2000 and March 2001. All areas of the system are experiencing increasing pressures, manifesting itself in long waiting times for patients, stressed and overworked staff, hospital wards running close to capacity limits, and fewer elective (planned) admissions as the hospitals struggle to cope with the workload generated by the emergencies.

This problem is by no means confined to Nottingham. Recent reports by the UK Audit Commission ${ }^{2,3}$ have highlighted the fact that despite some improvements in a few areas, by and large in England and Wales A\&E waiting times, both to see a doctor and also to be admitted to hospital, have increased steadily since 1996. In 1996, about $72 \%$ of all patients were seen within $1 \mathrm{~h}$ of arrival in A\&E, but this had fallen to about $53 \%$ by 2000 . In 1996, about $89 \%$ of patients who needed in-patient care were admitted

Table 1 Increases on the previous year's emergency admissions to Nottingham hospitals

\begin{tabular}{lcc}
\hline & $1999-2000$ & $2000-2001$ \\
\hline QMC & $4.7 \%$ & $5.6 \%$ \\
NCH & $2.3 \%$ & $10.1 \%$ \\
\hline
\end{tabular}

within $4 \mathrm{~h}$, but this had fallen to $76 \%$ by $2000 .^{3}$ There is also significant unexplained variation between departments; there is a tendency for larger departments to have longer waits, but the association is weak - in fact, the Audit Commission found that the most significant single factor influencing waiting time was location in or out of London. Since 1996 patient numbers attending $A \& E$ in England and Wales have increased by $1 \%$ per annum, whereas the number of nurses has remained roughly the same. ${ }^{3}$ Nurse workloads vary widely between departments (from less than 1000 to more than 2000 patients per nurse per annum). However, the total number of doctors has increased by $10 \%$ since 1998 , especially in the more senior 'non-consultant career grades' 3 This is not, therefore, a simple problem of supply and demand, neither is it a straightforward issue of maximizing the throughput of a production system. The Audit Commission report suggests that long waiting times are caused by 'a host of managerial and organizational differences as much by resources and staff levels'.

The ECOD project grew out of an earlier initiative for winter crisis planning in Nottingham, when it became apparent that the 'crisis' was a chronic state of affairs rather than a temporary acute problem. A Steering Committee was set up early in 2001, containing representatives from all the health-care providers in Nottingham, and a Project Team formed, chaired by a local GP and including a full-time project manager. The aim of the ECOD project was to develop a new Local Services Framework for emergency care, which would form the basis of future strategy in Nottingham. The University of Southampton team began work in August 2001 and completed the research project in April 2002. There were four key research questions to be addressed:

- How is the emergency/on demand system currently configured and what organisational systems, processes and responsibilities support it?

- What characteristics of demand, demand management and patient flows can be identified from retrospective analysis of activity data, observational data and the views of key informants?

- How should the emergency care/on demand system be developed to respond to health policy and local needs, and what are the economic implications?

- To what extent do community preferences account for current use of the emergency care/on demand system and how can they inform its development?

The research project itself has been described elsewhere ${ }^{4}$ and comprised several strands, involving a literature review, activity data collection and analysis, stakeholder interviews and a patient preference survey. ${ }^{5}$ In this paper, we shall describe the contribution of two simulation models, a system dynamics model of the whole system and a smaller discreteevent simulation model of the A\&E department, in helping to provide answers to the first three of these four questions. 


\section{Choice of modelling approach}

An early decision was whether to adopt a discrete or continuous simulation approach. Historically, there have been very few examples in the health-care modelling literature of discrete-event simulation (DES) models for very large populations. ${ }^{6}$ This is essentially because a DES model with over a hundred thousand entities (ie patients) would require a vast amount of computer memory and would be very slow to run. Despite advances in computing power and the use of efficient queue sorting techniques, DES models are still time-consuming to run, since every individual patient's 'life history' is modelled. Moreover, multiple iterations must be performed to account for random variation. These problems do not arise for system dynamics, which is not stochastic and does not model patients at the individual level.

System dynamics (SD) is an analytical modelling approach originally developed by Jay Forrester ${ }^{7,8}$ in the 1960s in his work on 'industrial dynamics'. SD combines qualitative and quantitative aspects, and aims to enhance understanding of a system and the relationships between different system components. The concepts of feedback and causal effects are important in SD. Surprisingly, perhaps, there have been relatively few applications of SD in healthcare, compared with the vast number of DES applications. ${ }^{9}$ Dangerfield and Roberts' SD models for HIV/AIDS are well known. ${ }^{10}$ A more recent example is Townshend and Turner's model ${ }^{11}$ for screening for Chlamydia, a major cause of infertility. Townshend and Turner chose SD partly because the populations in this model were large, and partly because SD could incorporate the feedback effects due to re-infection of treated people, and the reduction in the prevalence of Chlamydia after screening. Wolstenholme's model ${ }^{12}$ was one of the first well-known applications of qualitative SD in healthcare, and showed that an (unintended) effect of the UK's 1993 Community Care Act would in fact be to increase social service spending. Another well-known SD example, focusing on A\&E, is David Lane's model, ${ }^{13}$ which was designed to explore the relationships between waiting times in $A \& E$ and bed closures. The argument was that bed reductions led to cancelled elective admissions and this led to more people presenting in A\&E, partly as a direct result of the deterioration in their health and partly as a behavioural response by doctors wishing to get their patients admitted 'by the back door'. The key finding was that the major impact of bed shortages was not on emergency admissions, but was felt first on elective admissions, so that using A\&E waiting times to measure the effect of bed shortages was misleading.

In the Nottingham study, we were dealing with a very large, complex system involving a population of over 600000 potential patients. Furthermore, we considered that although the specific pathways followed by individual patients were of interest, they were of less importance than understanding the major flows of people through the 'front doors' to the NHS, and gaining insight into the general structure of the system and the relationships between its component parts. The problems experienced in $\mathrm{A} \& \mathrm{E}$, for example, were not principally felt to be due to high variability in casemix or staffing levels, but more to the sheer volume of demand and consequent pressure on resources. Finally, we were less concerned with the waiting times of individual people than with the general flow of patients through the system, in order to identify bottlenecks. Thus, system dynamics was chosen as our modelling approach and we used both qualitative and quantitative aspects.

\section{Phases of model development}

\section{Qualitative phase}

The aim of this phase was to develop understanding of the ECOD system, not only by the research team but also by the stakeholders in the system. It was not merely a preliminary stage to the quantitative modelling, but was important in its own right. Many useful insights were gained as a result of the development of the conceptual map and through the interview process.

During August 2001, the research team made an orientation visit to Nottingham, in which a first-pass 'conceptual map' of the system was drawn up. The first stage of this was to list the 'front doors' or access points to the health-care system, and then to expand this to show the connections between these access points and the other parts of the system. A highly simplified version of this map is shown in Figure 1. The diamond on the far left-hand side represents the patient's initial decision to contact the health service. The conceptual map was printed on A3 paper and used as the basis of 30 semi-structured interviews with key individuals from all the main health-care providers, together with patient representatives. These interviews took place between September and November 2001. These 'stakeholders' were selected in consultation with the Project Team and the Steering Committee. The participants' initial agreement was sought by telephone and they were sent a copy of the interview schedule in advance, to allow time for preparation. All but one of the interviews were taperecorded and later transcribed for analysis.

During the interviews, participants were asked about their work roles and the capacity they felt they had to influence the interface between their part of the system and other components. This led on to a discussion of the interfaces between components and the factors that might influence patient flows through the system. Participants were asked to draw on the map to show these influences and to annotate or alter the map in any way they felt appropriate. As a result a final agreed version of the map was derived, which was later 


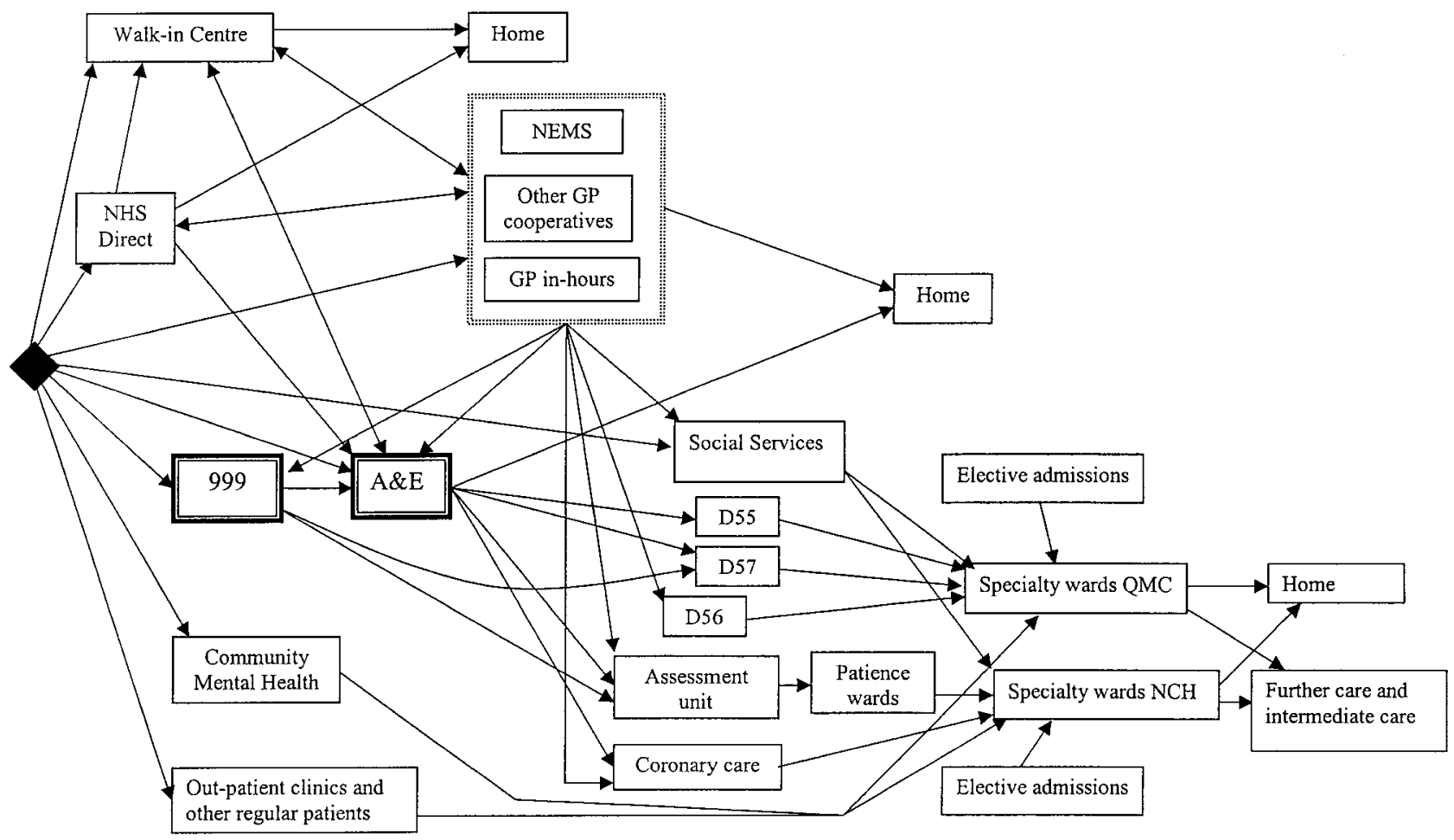

Figure 1 Simplified 'conceptual map' of the emergency health-care system in Nottingham. D55, D56 and D57 are acute admission wards in QMC.

used as the basis for a quantitative computer model of the system using the software STELLA. ${ }^{14}$

\section{Quantitative phase}

The aim of this phase was to facilitate experimentation with various potential changes in service configurations and demand rates. A stock-flow modelling approach was used, where stocks represented accumulations of patients (eg, waiting to see a GP, waiting for treatment in $A \& E$, or occupying a bed in an acute admission ward) and the flows were the admission, transfer, treatment and discharge rates.

STELLA (also known as ithink) is a user-friendly package with a drag-and-drop user interface which allows the modeller to develop the model without the need for programming. The layout of the computer screen followed that of the conceptual map, so that the top half represented the primary care sector (in-hours and out-of-hours GP surgeries, NHS Direct and the Walk-in Centre) and the bottom half represented the secondary sector (the two main hospitals). In the middle was the Ambulance Service, Social Services and the A\&E Department. We did not attempt to model every single hospital ward, but just the admissions wards. Onward transfers to the main specialty wards after a stay in an admissions ward were not modelled in detail, as we were concerned principally with patient flows within the emergency system. STELLA uses submodels to make the model more transparent by concealing detail, and these were used for each of the sectors. We used a single submodel to represent all the main specialty wards in each hospital.

For example, Figure 2 shows the internal flows within the NHS Direct submodel. As a result of the integration of NHS Direct with the GP out-of-hours cooperative NEMS, patients may arrive from two sources, depending on the time of day. During surgery hours, every caller is an NHS Direct 'self-referral', but outside surgery hours some patients will be calling NHS Direct in order to contact NEMS. Inside the NHS Direct submodel, patients are routed on to one of seven possible destinations: the Walk-in Centre, the ambulance service, NEMS, the commercial out-of-hours GP deputizing service HealthCall, the in-hours GP surgery, A\&E, or 'home', meaning given self-care advice over the telephone. In this and the other front door submodels, we were not concerned with patient waiting times, but rather with the proportions of patients routed on to other providers. The outflows from the NHS Direct submodel become inflows to the seven destination sectors.

Other submodels, for example, the Assessment Unit (the acute admissions unit at $\mathrm{NCH}$ ) contain information about the bed capacity and the influence of various factors, such as patient age, bed occupancy rates and day of week on the length of stay. The model thus allowed a top-level, global view of the whole system, with the capability of drilling down to lower levels of detail in specific areas if necessary. 


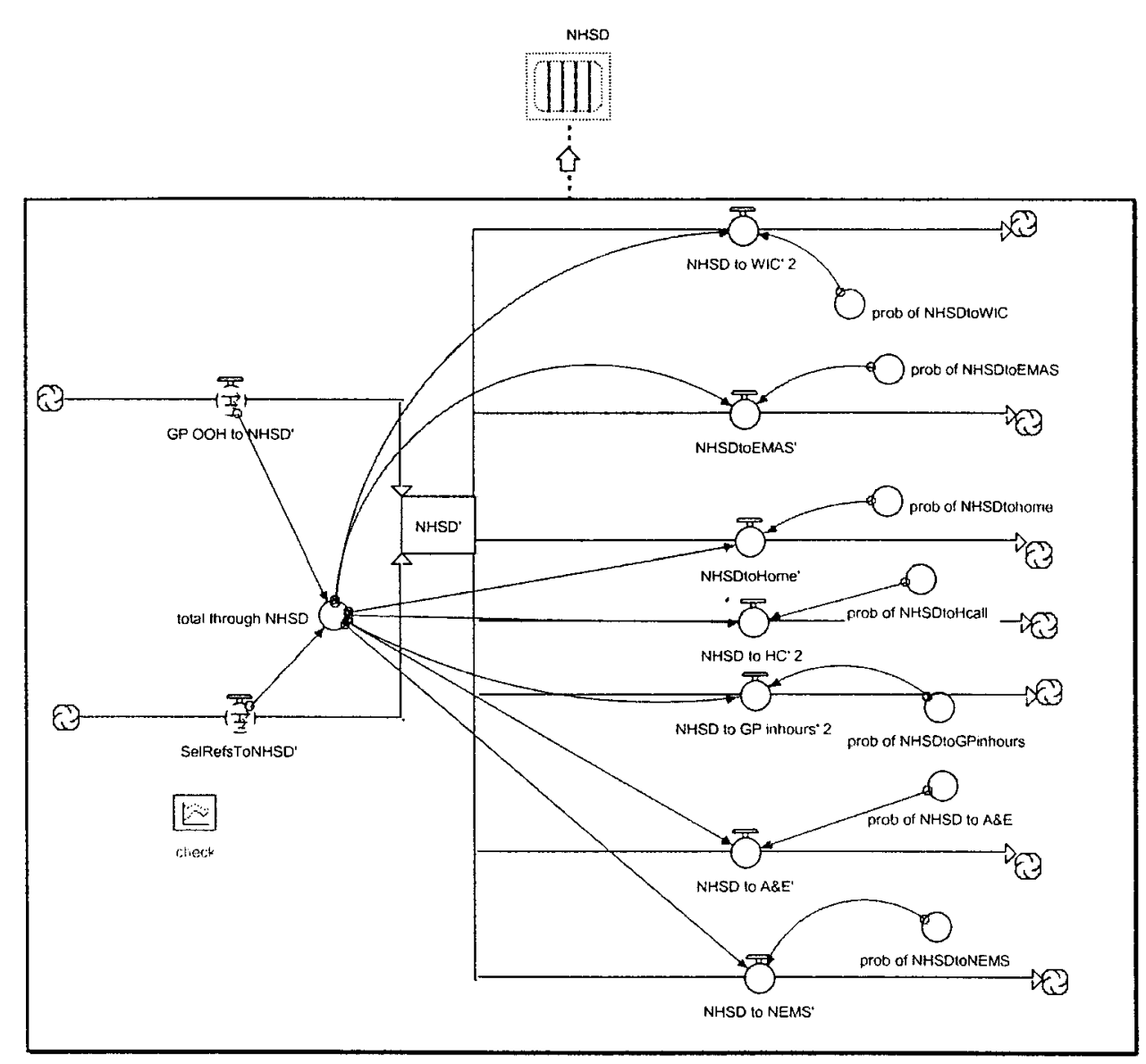

Figure 2 The NHS Direct submodel of the STELLA model.

The STELLA model was populated with data for the year April 2000-March 2001, obtained from the various providers in Nottingham. These comprised the patient arrivals, broken down where possible by hour and day, sex and age band and where appropriate category of urgency; the source of the arrival, and the destination (eg emergency hospital admission, discharge home or elsewhere). Hospital length of stay data were derived from the Hospital Episode Statistics provided by the Department of Health. ${ }^{15}$ This enabled flow balance cross-checking to be carried out, although the quality and level of detail of the data were variable. The outflow to B reported by A must equal the inflow from A reported by B. Unfortunately, no system-wide data were available for the in-hours GP sector, although we collected prospective data for a single week from four individual practices. We therefore had to rely solely on the hospital data regarding GP admissions, which essentially produced a discontinuity in the model for this particular flow.

As in all stock-flow systems, the contents of each stock or reservoir are updated at regular intervals by solving a set of difference equations representing the inflows and outflows from that stock. The choice of the time-step $\mathrm{d} t$ was difficult, given the wide range in activity durations (some only took minutes, others took days or even weeks), but we chose a value of $\mathrm{d} t$ equal to $2.4 \mathrm{~h}$ ( 0.1 days, $144 \mathrm{~min})$. STELLA presents results in the form of graphs and tables, but most of our output was exported to Excel for analysis and presentation purposes. The output included the throughput of each 'front door' and the occupancy rates of each of the wards and hospital departments. STELLA allows the user to break down stocks and flows into subscripted arrays; for example, to classify patients by age, but it is not possible to combine an arrayed model with submodels. We decided that the benefits of using submodels outweighed the benefits of arrays, as we were able to account for age where necessary by using extra stocks, flows and auxiliary variables.

\section{Model validation}

The validation of SD models is a thorny topic. It has been argued $^{16}$ that validation of qualitative models should be carried out with the client as an ongoing dialogue during the model-building process, and is essentially a 'white box' process ${ }^{17}$ where the client knows, understands and trusts the internal structure of the model. The aim of qualitative models is not to produce point estimates or to 'optimize', but to gain insights into the system and learn about the way it behaves. On the other hand, quantitative SD models can be 
Daily Bed Occupancy Rates, Nottingham City Hospital

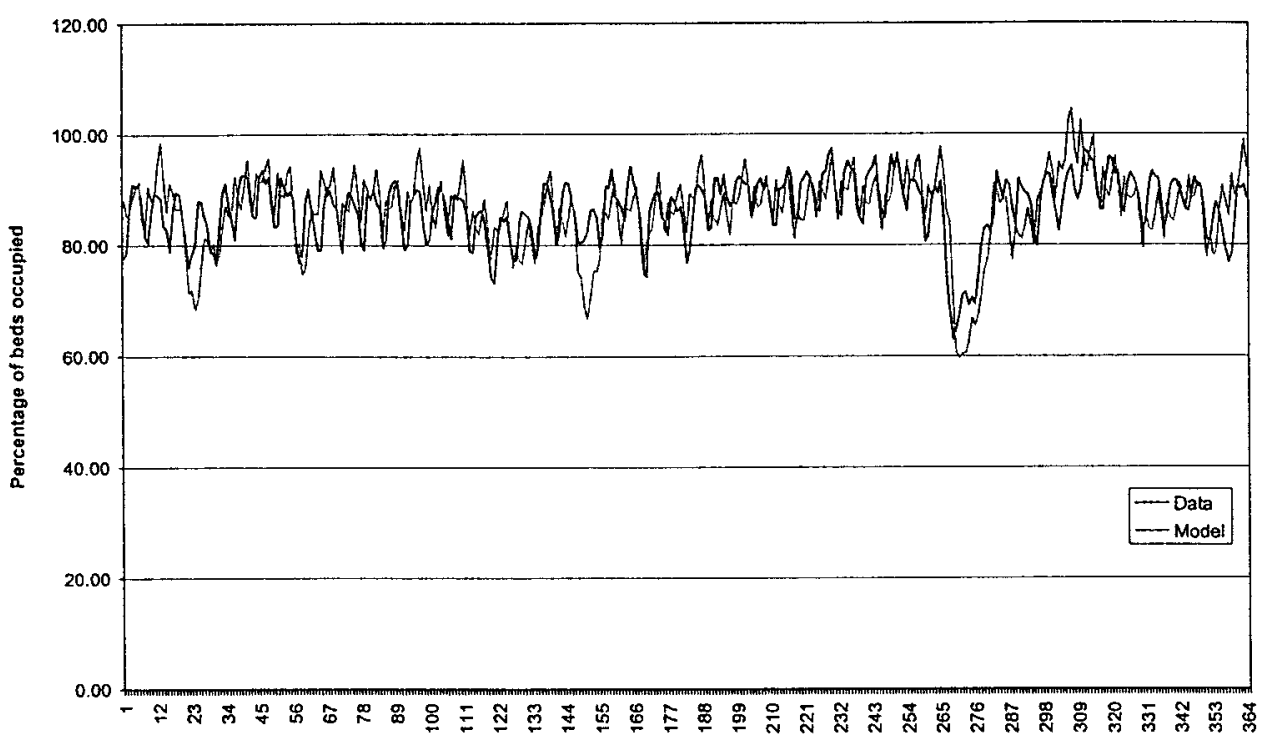

Figure 3 Model validation, using total daily bed occupancy of NCH for 2000-2001.

validated, in the same way as any other numerical simulation model, ${ }^{16}$ by a 'black box' process ${ }^{17}$ where emphasis is not on the model structure, but on the output it produces.

In our case, we used both approaches. We developed the model in close collaboration with the Steering Group during frequent visits to Nottingham. In addition to the inflowoutflow balance checking described above, we carried out 'black box' validation by running the model for the period April 2000 to March 2001, using the known arrivals data, and comparing the model output with real-life system performance data which had not been used in the construction of the model. For example, we used the total daily bed occupancy (formerly known as 'midnight bed state' data) supplied by the hospitals' Information Management and Technology Departments, and compared this with the corresponding model output by aggregating all the individual ward bed occupancies (see Figure 3), to give confidence that the model was producing sensible output. Similar plots were obtained for other output parameters such as individual ward occupancies.

\section{Scenario testing: model results}

The Steering Committee suggested a range of scenarios for testing, based on the comments of the interview participants. For example, it was suggested that GPs are admitting some patients as emergencies in order to get investigations carried out, which could equally well be performed as day cases or even outpatients, because of the lack of suitable facilities. This is a similar behavioural response to that identified in Lane's study in London. ${ }^{12}$ A community Diagnostic and Treatment Centre (DTC) where such tests could be carried out could therefore prevent many 'unnecessary' admissions.

A planning horizon of 5 years was used. The scenarios included the 'Doomsday scenario' (maintaining current growth in demand with no additional resources) and a variety of possible alternatives, including:

- 3\% year-on-year growth in GP referrals for planned admissions,

- reduced emergency admissions for certain patient groups (eg the elderly or people with respiratory disease), for example, by the use of a DTC or other community initiatives,

- earlier discharge of the elderly to nursing homes,

- the effects of 'streaming' in the A\&E department, that is, separate resources for certain patient groups.

The key outputs from the system map and STELLA model were initially, the insights gained into different parts of the system by people seeing it as a whole for the first time. Simple influence diagrams describing parts of the system were found to be a powerful tool in stimulating debate. For example, it could be argued that long waiting times in $A \& E$ are not necessarily always a bad thing, in that the expectation of a long wait might discourage 'inappropriate' attenders and lead them to seek help elsewhere, perhaps in the Walk-in Centre or by phoning NHS Direct. The patient preference study ${ }^{5}$ showed that many people were indeed deterred by the prospect of a long wait and would only go to $A \& E$ if they felt they really needed to be there (eg, if they thought they needed an X-ray). Thus, inadequately thoughtout initiatives designed to reduce waiting times in $\mathrm{A} \& \mathrm{E}$ might actually turn out to be counterproductive. 
Table 2 Average percentage occupancy of both hospitals, assuming a sustained year-on-year decrease of 3\% in emergency admissions of people aged over 60 years

\begin{tabular}{lll}
\hline & $N C H$ & $Q M C$ \\
\hline 'Status quo' & 86.7 & 84.7 \\
$2000-2001$ & 85 & 83 \\
$2001-2002$ & 84 & 82 \\
$2002-2003$ & 83 & 81 \\
$2003-2004$ & 82 & 80 \\
$2004-2005$ & 80 & 79 \\
\hline
\end{tabular}

The first main result from the scenarios was that the system is currently operating dangerously close to capacity. This reinforced the message coming across from many of the stakeholder interviews. The model showed that if growth in emergency admissions continues at the current rate, both hospitals will see a significant decrease in the number of elective admissions within 4 years. City Hospital, for example, could expect to see at least a $25 \%$ drop in elective admissions by 2005 (from 1100 per month in 2000-2001, to 700 in 2004-2005). The scenario where planned GP admissions were constrained to increase by $3 \%$ per annum was even worse, with average bed occupancies exceeding $100 \%$ by 2005 (assuming no additional resources).

The model also showed how small changes to one part of the system can have a considerable impact elsewhere in the system. For example, the effect on average total bed occupancy of sending $3 \%$ of patients aged over 60 to a DTC instead of admitting them is shown in Table 2 . If a $3 \%$ reduction were maintained year-on-year for 5 years, a significant decrease in total occupancy could be achieved. The bed occupancy target for 2004 set by Government ${ }^{18}$ is $82 \%$ and the current figures for QMC and $\mathrm{NCH}$ are 84.7 and $86.7 \%$, respectively. Bagust et $a l^{19}$ have used DES to show that it is risky to have average occupancy figures higher than $85 \%$.

Interventions targeted at patients with specific health problems, such as respiratory conditions or ill-defined diagnoses, did have an effect, although it was not large. Reducing emergency admissions for patients with respiratory problems (by $20 \%$ per annum year on year for four years) reduced overall bed occupancy by approximately $2 \%$, a small annual effect. However, the seasonal nature of the reductions in admissions gave increased benefits, as the January peak in occupancy was more significantly reduced relative to other months.

Interventions aimed at preventing 3 or $6 \%$ emergency admissions of patients over 60 years of age made a substantial difference in the model. Even without assuming any decrease in average length of stay, bed occupancy in both hospitals was reduced by $1 \%$ per annum over the 5 year duration. This is to be expected since people in this age group comprise around about half of all emergency admissions.
We evaluated the effect of early discharge for patients admitted as emergencies, who were subsequently discharged to nursing homes. Despite the common perception of 'bedblockers', discharging these patients 2 days early made hardly any difference to overall occupancy rates, and there appeared to be surprisingly little potential for improvement in this area. We also investigated the effects of 7-day-a-week discharging from hospital. This showed a small decrease in occupancy, although care needs to be taken in interpreting the model results here, since the admission days for elective patients are currently planned to accommodate weekday discharging. However, some benefit might still be achieved. Overall, though, the model showed that the effects of discharging these people earlier were minimal compared with the effects of keeping them out of hospital in the first place.

\section{The A\&E model}

We were asked to investigate the Government suggestion ${ }^{18}$ that waiting times in A\&E could be reduced by the provision of 'fast track' systems for minor injuries or illnesses. Patients streamed in this way would have their own waiting area and dedicated staff, and would not share resources with other A\&E patients. Streaming patients appears counterintuitive from a queueing theory perspective, until we take into account the fact that different categories of patients have different acceptable waiting times and hence different targets. Thus although some patients may have to wait for longer, their waiting time could still be within acceptable limits. For a description of how such a system might be implemented in practice, see Cooke et al. ${ }^{20}$

Unfortunately, system dynamics does not ideally lend itself to narrowly focussed systems involving resourceconstrained queueing networks. For problems requiring this level of individual detail, discrete-event simulation is the method of choice. ${ }^{6}$ A separate, very simple DES model for A\&E was therefore rapidly developed using the software Simul $8^{21}$ and was populated with patient arrival and staff resource level data from the A\&E department at QMC. Activity duration data were derived from the literature ${ }^{2,22}$ as there was no time to gather primary data in this study.

On arrival in A\&E, patients are initially prioritised into five urgency categories, where 1 denotes life-threatening conditions and 5 denotes minor injury or illness. This process is called triage. Category 1 patients are always seen immediately, but lower category patients are seen in priority order as resources permit and may have to wait. We investigated the streaming of minor cases (triage categories 4 and 5). We found that the permanent streaming of minor injuries was not an efficient use of clinical resources. Improvements were observed for the less urgent patients, but these were at the expense of patients in categories 2 and 3. The results for this scenario are shown in Table 3. 
Table 3 Results from the A\&E streaming model

\begin{tabular}{llc}
\hline & Performance indicator & Without streaming \\
\hline Shared doctors & $\%$ Utilisation & 70 \\
Stream doctors & $\%$ Utilisation & - \\
Cat 2 treatment & $\%$ Queued less than $10 \mathrm{~min}$ & 96 \\
Cat 3 treatment & $\%$ Queued less than $60 \mathrm{~min}$ & 99 \\
Cat 4 treatment & $\%$ Queued less than $120 \mathrm{~min}$ & 87 \\
Cat 5 treatment & $\%$ Queued less than 240 min & 83 \\
\hline
\end{tabular}

A flexible system appears to be required in which streaming is only triggered when waiting times reach a certain threshold. This is in accordance with the findings of Cooke et al..$^{20} \mathrm{~A}$ compromise solution for Nottingham may be to dedicate one doctor to the fast track patients, and have a second doctor on standby to join the first doctor if there is a sudden rush of minor cases. Other solutions may well involve the use of Emergency Nurse Practitioners to deal with less serious patients, releasing doctors to work with the more serious cases. Further simulation modelling work could help here, for example, in determining the threshold for initiating streaming.

\section{Discussion}

Both the qualitative and quantitative aspects of the system dynamics approach proved to be very useful in this project. The conceptual map provided a helpful structure around which to base the stakeholder interviews. Many participants commented on the value of seeing the whole system in its entirety, often for the first time, and on the insights they gained about how other parts of the system related to the part with which they were familiar. Although causal loop (influence) diagrams were not constructed for the entire system, they were used to gain insight into the behaviour of parts of the system.

The STELLA model was useful on two levels-firstly, naturally, for investigating specific scenarios in terms of patient flows and bottlenecks, but secondly (and perhaps equally importantly) as a device for provoking and facilitating discussion and comment. Interestingly, although the Nottingham Steering Group were initially fascinated by the computer model and the visual and numerical output, they readily accepted the idea that the model gave an indication of the relative effects of different interventions rather than mathematically precise forecasts or point predictions. They were very keen to suggest alternative scenarios for testing, arising from the findings of earlier runs of the model.

This study was conducted in partnership with a health and social care community in Nottingham already committed to the concept of partnership working and the need for a "whole systems approach' to development. The process and findings of this independent enquiry appear to have contributed to sustained local efforts to find better solutions for the benefit of the people of Nottingham, and have informed the articulation of a local service framework for emergency care. The SD model we have constructed has the potential to evaluate the impact of the real system developments that are now envisaged in Nottingham.

The approach adopted in Nottingham could easily be applied elsewhere. The process of stakeholder interviews and the development of a conceptual system map is a generic one which could be used anywhere. Emergency and on-demand health-care systems in different geographical areas may differ slightly but will share many common features, and the STELLA model for Nottingham could easily be reconfigured for a different location and repopulated with the appropriate data. Much of the necessary data are now routinely collected by Trusts for management purposes. We believe this approach could make a substantial, practical contribution to the improvement of emergency health-care delivery.

Acknowledgements - We thank the health and social care staff who assisted us in the conduct of this study and Dr Stephen Shortt, Mr James Scott, Mr John MacDonald, Dr Doug Black and the local steering committee for their contribution. We also thank Mr Steve Baxter and Mr Shaun Leah for their assistance in providing data. Nottingham Health Authority funded the project but the views expressed in the paper are those of the authors alone.

\section{References}

1 http://www.nottinghm-ha.trent.nhs.uk, downloaded Oct 21, 2002.

2 Audit Commission (1996). By Accident or Design: Improving $A \& E$ Services in England and Wales. HMSO: London.

3 Audit Commission (2001). Accident and Emergency Acute Hospital Portfolio: Review of National Findings. Audit Commission: London.

4 Lattimer VA et al (2004). Reviewing emergency care systems I: insights from system dynamics modelling. Emerg Med J, In press.

5 Gerard K et al (2004). Reviewing emergency care systems II: measuring patient preferences using a discrete choice experiment. Emerg Med $J$, In press.

6 Brailsford SC and Hilton NA (2001). A comparison of discrete event simulation and system dynamics for modelling healthcare systems. In: Riley J (ed) Proceedings from ORAHS 2000, Glasgow, Scotland, pp 18-39. 
7 Forrester JW (1961). Industrial Dynamics. MIT Press: Cambridge, MA.

8 Forrester JW (1960). The impact of feedback control concepts on the management sciences. In: Collected Papers of J.W. Forrester (1975 collection), Wright-Allen Press: Cambridge, MA, pp 45-60.

9 Jun JB, Jacobson SH and Swisher JR (1999). Application of discrete-event simulation in health care clinics: a survey. $J \mathrm{Opl}$ Res Soc 50: 109-123.

10 Dangerfield BC and Roberts CA (1990). Modelling the epidemiological consequences of HIV infection and AIDS: a contribution from Operational Research. J Opl Res Soc 41: 273-289.

11 Townshend JRP and Turner HS (2000). Analysing the effect of Chlamydia screening. J Opl Res Soc 51: 812-824.

12 Wolstenholme EF (1993). A case study in community care using systems thinking. J Opl Res Soc 44: 925-934.

13 Lane DC, Monefeldt C and Rosenhead JV (2000). Looking in the wrong place for healthcare improvements: A system dynamics study of an accident and emergency department. J Opl Res Soc 51: 518-531.

14 STELLA, High Performance Systems, 145 Lyme Road, Hanover, NH.
15 Department of Health (2002). Hospital Episode Statistics. Department of Health: London.

16 Lane DC (2000). You just don't understand me: modes of failure and success in the discourse between system dynamics and discrete event simulation, Working paper no. LSEOR 00.34, London School of Economics.

17 Pidd M (1998). Computer Simulation in Management Science, 4th edn. Wiley: Chichester.

18 Department of Health (2001). Reforming Emergency Care, Downloadable from www.doh.gov.uk/capacityplanning/reformfirststeps.htm.

19 Bagust A et al (1999). Dynamics of bed use in accommodating emergency admissions: stochastic simulation model. BMJ 319: $155-159$.

20 Cooke MW, Wilson S and Pearson S (2002). The effect of a separate stream for minor injuries on accident and emergency department waiting times. Emerg Med $J$ 19: 28-30.

21 Simul8, www.Simul8.com.

22 Shrimpling M (2002). Redesigning triage to reduce waiting times. Emerg Nurse 10: 34-37.

Received April 2003; accepted October 2003 